\title{
Análisis
}

\section{Kube: una nueva forma de gestionar proyectos}

\section{Introducción}

El portal corporativo de $M I K$ es una solución web que engloba, bajo una misma plataforma, la imagen corporativa de $M I K S$. Co$o p$., la gestión documental de todos sus proyectos y la Comunidad virtual de investigación en gestión (denominada Kube) que constituye un espacio de colaboración y experimentación revolucionario donde se pueden generar, gestionar y coordinar proyectos compartidos por múltiples organizaciones geográficamente dispersas. Concebimos Kube como una nueva forma de gestionar proyectos ya que, además de su coordinación básica, facilita tareas hasta ahora dispersas en los sistemas existentes como la gestión por competencias, nuevas tecnologías para la colaboración, traducción y volcado de la información a distintos estándares europeos, estatales, etc.; capacidad para detectar, retener y reutilizar el conocimiento tácito que generan los distintos proyectos, tales como las buenas practicas, experiencias, contactos, etc. Kube soporta bajo una misma plataforma las 7 áreas clave del ciclo de vida de un proyecto como son: generación de nuevas ideas, propuestas de proyectos, adhesiones, formación de equipos, presentación y oferta de proyectos, formatos administrativos y workflow de proyectos, cada una de las cuales desarrollaremos más en detalle. Este nuevo espacio de gestión e innovación surge del acuerdo alcanzado entre Mondragón Innovation \& Knowledge (MIK S. Coop.), centro de investigación en gestión del conocimiento y de experimentación en modelos y herramientas avanzadas de

\section{Por Javier Fínez y Emilio González}

dirección y management, perteneciente al grupo MCC y Ever Documéntica empresa filial del grupo Ever-Team, considerado el primer grupo francés de desarrolladores de soluciones de gestión de documentos y de contenidos con una base instalada superior a 2.000 clientes. El sistema de explotación que lo soporta se denomina EverSuite, que es un conjunto de servicios web desarrollados en Java en el entorno del estándar J2EE.

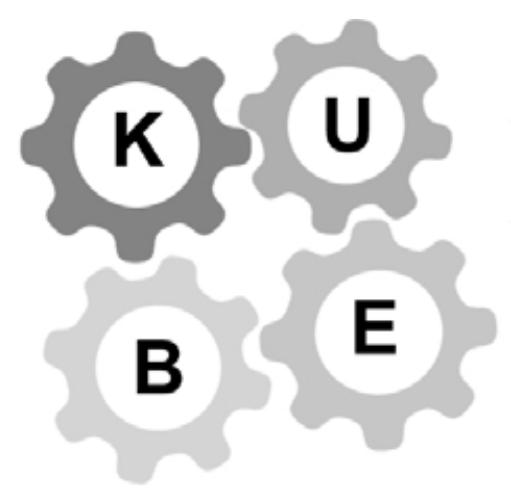

\section{Funcionalidades}

Empecemos por destacar un hecho': actualmente utilizamos de forma eficiente únicamente un $15 \%$ de la información que poseemos en nuestra organización para desarrollar nuestros proyectos. El resto está sin "estructurar": páginas web, e-mails, buenas prácticas, bibliografía y mucha más información que se cuela a diario en nuestras oficinas o está disponible "out there". El reto para nuestras organizaciones es asegurar que nuestros trabajadores del conocimiento poseen un método efectivo de acceder rápidamente a esa información que en algunos casos es vital.

El portal corporativo de $M I K$ tiene su ubicación y centro virtual en dicha organización y está abierto a toda persona, colectivo, insti- tución o empresa deseosa de compartir, adquirir y gestionar ideas, conocimientos y proyectos. En la figura 1, vemos los tres ejes básicos sobre los que se estructura el portal corporativo:

-Imagen corporativa. Una parte mínima de este conocimiento estructurado se encuentra en la información de tipo institucional que sobre $M I K$ se ofrece en esta área al público en general. Además de reflejar nuestras señas de identidad, visión y valores, áreas actuales de investigación, etc. una de las principales innovaciones incorporadas es la posibilidad de editar el contenido de estas páginas por cualquiera de los miembros de $M I K$ sin necesidad de poseer conocimiento previo de html o ASP y poder introducir cambios en sus C. V., proyectos que lideran, áreas de interés etc.

- Gestión documental. Constituye el segundo pilar. Surgida con la idea de que sea un área evidentemente dinámica, permite gestionar apartados de frecuente actualización en una web como son los referentes a artículos, publicaciones, noticias de interés, etc. Así, admite que alguien externo realice colaboraciones en forma de artículos o comentarios a publicaciones divulgadas en nuestro portal y, previo paso de nuestro filtro de moderación, permitir incluirlas en dichos espacios. Permite, de forma complementaria, la suscripción a noticias, calendario de eventos, foros de intercambio, etc.

Además de esta área de libre acceso, hemos desarrollado un área restringida que denominamos intranet colaborativa, que es el punto 
de encuentro de los investigadores de $M I K$, está organizado por proyectos y áreas de trabajo y su objeto es gestionar eficientemente toda la documentación que se genera en $M I K$ y que se pretende sea privada. Este espacio hace posible la creación de nuestras propias carpetas, insertar y crear documentos en múltiples formatos, posee la facilidad de calendario para convocar reuniones internas, tablón de anuncios internos, etc.

- Sin embargo, lo que otorga una dimensión hasta ahora desconocida a nuestro portal es la Сотиnidad virtual de investigación en gestión (Kube) que es la solución que permite optimizar la generación y coordinación de proyectos desde el punto de vista del conocimiento, de los equipos y de la estrategia sobre una plataforma colaborativa y dinámica desarrollada como fruto de la alianza entre $M I K$ y Ever Documéntica, experta en soluciones enterprise content management (ECM).

\section{Kube: una nueva forma de gestionar proyectos}

El objetivo marcado es crear un entorno de trabajo colaborativo, dinámico, seguro y capaz de reunir toda la información desestructurada de una organización, analizarla, categorizarla y diseminarla con el ánimo de mejorar la productividad y la toma de decisiones de los individuos mediante el acceso a la información que se necesita en el momento preciso. Esta nueva forma de interaccionar, de coordinarse y de trabajar, presenta grandes potencialidades de mejora a la hora de gestionar proyectos y ofrece la posibilidad de incorporar nuevas tecnologías de comunicación, facilitando la integración de los diferentes grupos de trabajo que conforman un proyecto. Kube soporta las 7 áreas clave del ciclo de vida de un proyecto:

\section{Generación de nuevas ideas.}

Es el lugar de la comunidad virtual donde los investigadores y colaboradores proponen, crean e intercambian ideas, de tal manera que como resultado de esta ósmosis surjan nuevas ideas y propuestas que a posteriori puedan transformarse en proyectos. Con el objetivo de otorgarle un carácter dinámico y creativo, se han diseñado cuatro entornos de actuación:

a. Herramientas de creatividad: es el espacio donde fluyen las ideas, que nos permite jugar entre la realidad y la ficción, que nos hace dudar de todo lo que sabemos pero que a la vez nos imbuye con nuevos conocimientos. En definitiva, es un semillero donde poder plantar nuestras ideas, contrastarlas con otros investigadores, ponerlas en tela de juicio, etc. Los facilitadores propuestos en este entorno son varios:

- Software de creatividad y generación de nuevas ideas desarrollado por MIK como Germinal ${ }^{2}$ y Roadmap Tool $^{3}$ que periódicamente vamos actualizando con nuevas versiones.

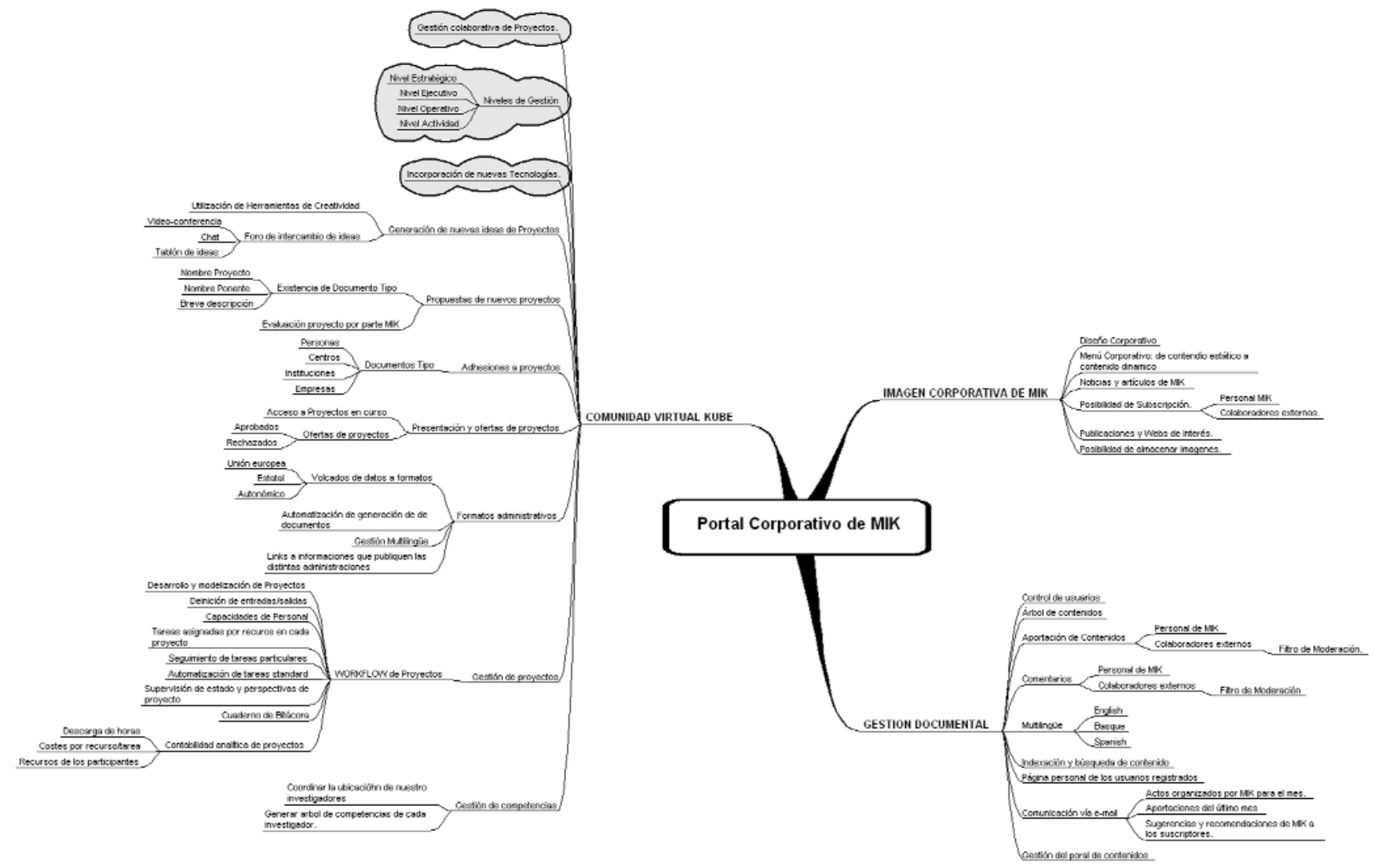

Figura 1: esquema completo del portal corporativo de MIK S. Coop 
—Posibilidad de incorporar, de manera sencilla, nuevas herramientas de creatividad e innovación.

- Almacenar links a páginas con software específico de generación de ideas.

- Proveer de referencias bibliográficas sobre técnicas de generación de ideas.

b. Foro de intercambio de ideas: se dispone en este caso de un tablón de ideas donde poder añadir sugerencias. Además se dispondrá de un chat y un servicio de calendario para poder convocar reuniones para discutir e intercambiar opiniones sobre diferentes temáticas, etc.

-Ideas locas: este foro permitirá registrar y hacer públicas todo tipo de propuestas y sugerencias por muy "descabelladas" que puedan parecer.

Para fomentar la participación, el acceso es libre a todas las personas que quieran participar tengan o no vinculación con $M I K$ y que deseen exponer sus ideas o inquietudes al resto de la comunidad.

\section{Propuesta de proyectos.}

Es el lugar de la comunidad virtual donde se recogerán todas las ideas que vayan surgiendo en el área anterior y que se considere que pueden tener cierta viabilidad y hayan suscitado cierto interés y apoyo entre la comunidad de investigadores. Aglutinando todo este conocimiento desestructurado (ideas, opiniones, apoyos, individuos y/o organizaciones interesadas) en un documento tipo, pasamos a su evaluación en $M I K$ en base a 3 criterios de viabilidad:

-Desde el punto de vista técnico.

-Desde una perspectiva económica.

—Del consorcio o posibles participantes.
Esta última valoración se realizará automáticamente mediante el módulo de gestión por competencias, que posee catalogados no sólo a los investigadores de $M I K$, sino también a otros colaboradores externos de acuerdo a sus áreas de interés o competencias, su tasa horaria, disponibilidad de calendario etc.

Una vez superada esta fase estas propuestas quedan registradas en dos categorías diferentes:

-Rechazados: proyectos evaluados y considerados no viables, pero aún así interesantes de cara al futuro.

-Viables: en este caso serían los ya analizados y considerados viables, por lo que son susceptibles de formar ofertas.

\section{Adhesiones a proyectos.}

Es el lugar de la comunidad virtual donde cualquier persona, centro, institución o empresa puede acceder para documentarse sobre los proyectos que $M I K$ ha considerado viables y, por tanto, se encuentran en fase de lanzamiento y necesitan ampliar su consorcio. La información disponible sobre objetivos, viabilidad, participación etc., les permitirá decidirse sobre la posibilidad de adherirse y registrarse en la plataforma para un determinado proyecto a través de un documento tipo que, a su vez, facilitará a $M I K$ realizar una primera evaluación sobre los grupos interesados.

\section{Formación de equipos.}

Una vez cerrado el plazo de adhesiones a una propuesta determinada, valiéndose como anteriormente del módulo de gestión por competencias, debemos decidir cuáles son válidas para sacar adelante la propuesta. Además permite realizar la búsqueda, entrar en contacto e invitar a instituciones, empresas o individuos que posean el conocimiento, perfil o experiencia que nos complementen aque- llas áreas que, a pesar de las adhesiones, no se encuentran suficientemente cubiertas.

De esta manera, en esta etapa modelamos un equipo multidisciplinar, perteneciente a distintas organizaciones posiblemente supranacionales y que abarca y posee capacidadsuficiente para desarrollar el proyecto concreto, y que vendrá definido ya a nivel de nombres concretos, tasas horarias, horas disponibles, etc.

Una vez hallada la combinación más ideal del consorcio, Kube permite a los administradores o líderes del proyecto el uso de herramientas colaborativas e interactivas para convocar a los investigadores a reuniones de primera toma de contacto del consorcio, kick-off meetings, etc. (figura 2).

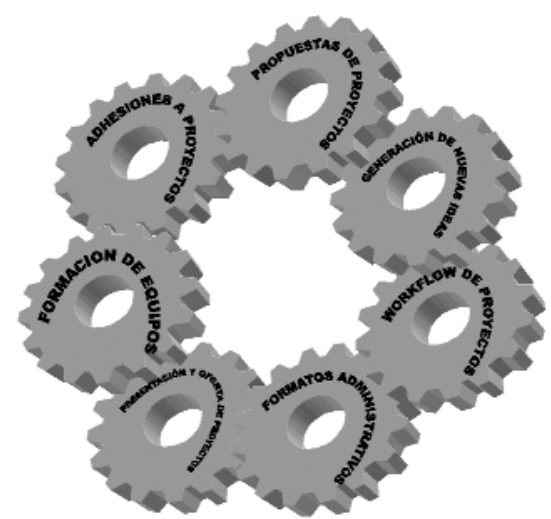

Figura 2: Kube: interacción entre las 7 áreas clave del ciclo de vida de un proyecto

\section{Presentación y oferta de proyectos.}

Este es el espacio en que, por fin, toda esa información desestructurada que hemos ido reuniendo en etapas anteriores cobra cohesión y se aproxima a lo que se llama un proyecto, ya que disponemos de todos los recursos necesarios para complementar, dar forma y volcar esta información sobre alguno de los documentos de oferta tipo que posee nuestra organización. De esta manera obtenemos una visión global, tanto de los aspectos cuantitativos (coste, duración, participantes) como de los 
cualitativos (objetivos, áreas de aplicación, impacto en el entorno, etc.).

Aquí encontramos otra de las ideas revolucionarias que definen Kube, pues en esta fase ya existen tiempos de ejecución, recursos humanos y materiales, objetivos, work packages, tareas, etc. que configuran un diseño de workflow incipiente pero que, en caso de ser aprobado el proyecto en alguna institución o empresa, puede ser volcado a un sistema de workflow visual y dinámico que permitirá operar sobre él, asignando y/o modificando tareas, plazos, presupuestos, personas etc. como veremos más adelante.

Dada la importancia que en esta fase de presentación de ofertas adquieren detalles tales como el formato, los plazos, los presupuestos, etc. esta área posee todas las facilidades necesarias para automatizar tareas hasta ahora muy tediosas como:

-Diagramas de Gantt en función de plazos, fechas y tareas del proyecto.

- Generar tablas de work packages y el contenido de los mismos.

- Localizar e insertar CVs de los integrantes del equipo de proyecto.

-Logotipos de las organizaciones e instituciones participantes.

-Presupuestos desglosados por años, work packages, otros apartados etc.

Desempeña también las veces de repositorio de ofertas en curso, rechazadas y aceptadas. Esta documentación servirá como fondo documental e histórico para complementar futuros proyectos.

\section{Formatos administrativos.}

Dado el carácter de centro tecnológico de $M I K$, las administra- ciones públicas tienen una gran relevancia a la hora de presentar nuestras ofertas. Básicamente son cuatro las administraciones con las que mantenemos un estrecho contacto: local, autonómica, estatal y europea. El gran problema al que nos enfrentamos en este tipo de concursos es que ya poseen su propio formato de presentación de ofertas, C. V. estandarizados, etc. Kube aborda estas cuestiones de dos maneras diferentes:

- Por una parte, tenemos un sistema de vigilancia inteligente que es capaz de detectar y avisar, a los suscriptores/investigadores que estén interesados cuando se produzcan cambios en sites de interés (BOE, Cordis, Bopv, etc.) que contienen tanto formularios de las distintas convocatorias como información relevante respecto a plazos, presupuestos, resoluciones, etc.

-Además, estos formatos administrativos son almacenados en Kube y, para cada uno de ellos, se procede a generar sus estructuras de datos, diseñar los formularios necesarios, analizar los diversos formatos y tipologías (tipos de letra, tablas de datos, etc.) del documento para tratar, de esta manera, de automatizar al máximo el volcado de información y generación de dicha documentación. Evidentemente este proceso debe ser desarrollado una sola vez para cada formato administrativo y por un usuario avanzado o administrador.

Por último, para hacer frente al multilingüismo que presentan las distintas administraciones incorporamos mecanismos de traducción al vuelo, teniendo la posibilidad de elegir entre varios traductores incorporados al sistema. El único problema de esta solución es que la fiabilidad de estas herramientas se ha demostrado a día de hoy bastante baja, aunque la plataforma está abierta para, de manera sencilla, incorporar otros nuevos a medida que sus prestaciones mejoren.

\section{Workflow de proyectos.}

En este momento nos encontramos en el escenario en que el proyecto ha sido aprobado por alguna institución o empresa. Su puesta en marcha y ejecución será tan sencilla como volcar el workflow incipiente estructurado en la etapa 5 a un formato visual, completamente dinámico que nos permite modificar plazos, recursos, asignaciones horarias, etc. y que haga posible visualizar en todo momento la situación real de los proyectos.

Este entorno será gestionado como un workflow dinámico al uso, donde tendremos entre otras las siguientes funcionalidades:

-Personalización y visibilidad adecuada al perfil.

—Desarrollo y modelización del proyecto.

-Definición de entradas y salidas de tareas.

- Seguimiento de tareas individualizadas.

- Contabilidad analítica (descargo de horas, coste de hora/recurso, etc.).

- Cuaderno de bitácora donde registrar el porqué de las decisiones.

-Etc.

Evidentemente, todos los individuos involucrados en el proyecto tendrán su lista de tareas, plazos y objetivos, así como unos permisos determinados que les permitirán acceder y/o modificar el flujo del proyecto, realizar descargo analítico de horas, contabilidad, control de versiones, "check in" y "check out" de entregables, documentos de trabajo etc. 


\section{Estrategias clave de Kube}

Para conseguir nuevas formas de pensamiento junto con nuevos entornos más creativos que nos ayuden a manejar un catálogo diferente de habilidades tales como gestión participativa, flexibilidad, ser agente facilitador de la innovación, etc., se ha llevado a cabo una verdadera revolución en el campo de las nuevas tecnologías, del intercambio y la creación de conocimiento, de la gestión por competencias, etc. He aquí algunas de las aportaciones de Kube que facilitan el desarrollo del trabajo comunitario y crean nuevas formas de interactuar y difundir el conocimiento.

1. Incorporación de nuevas tecnologías (Wall Display $)$.

La infraestructura tecnológica subyacente de Kube es modular y escalable siguiendo el estándar $J 2 E E$, permitiendo la integración con nuevas herramientas que vayan surgiendo en el ámbito de la creatividad, la interactividad, las nuevas tecnologías, etc., pudiendo además integrar mediante webservices funcionalidades de herramientas que abracen el estándar .net.

En línea con la integración de herramientas tecnológicas de última generación, se encuentra el Wall Display de $3 M$ que es una pantalla táctil que provee múltiples funcionalidades añadidas a la videoconferencia como: transmisión de imágenes en movimiento, animación, gráficos y conectividad instantánea con prácticamente cualquier tipo de sistema multimedia, pizarra de trabajo colaborativo, transferencia y captura de ficheros, etc.

La clave es la interactividad: la integración de esta herramienta hace posible a los miembros de la comunidad modificar la información sobre la pantalla para que el resto de investigadores conectados pue-

dan ver los cambios sobre la marcha, capturando de forma digital la información para reproducirla y distribuirla cuando sea necesario, estimulando de esta manera la creatividad, el brainstorming y el contacto casi cara a cara y en tiempo real con el resto de integrantes del equipo.

\section{Niveles de gestión.}

Es evidente, que se hace necesario gestionar de algún modo el acceso a la información existente en el sistema por motivos de seguridad, eficiencia y personalización de contenidos. Para proveer dicha funcionalidad se ha diseñado un sistema de niveles de gestión de acceso personalizados para cada grupo de investigadores, de tal manera que les permita ver sólo la información que les interesa y en el formato más eficiente para el desarrollo de su trabajo. De esta forma hemos dividido el sistema en cuatro niveles, dependiendo de cada perfil de usuario (figura 3 ):

'Transformación de aportaciones en proyectos

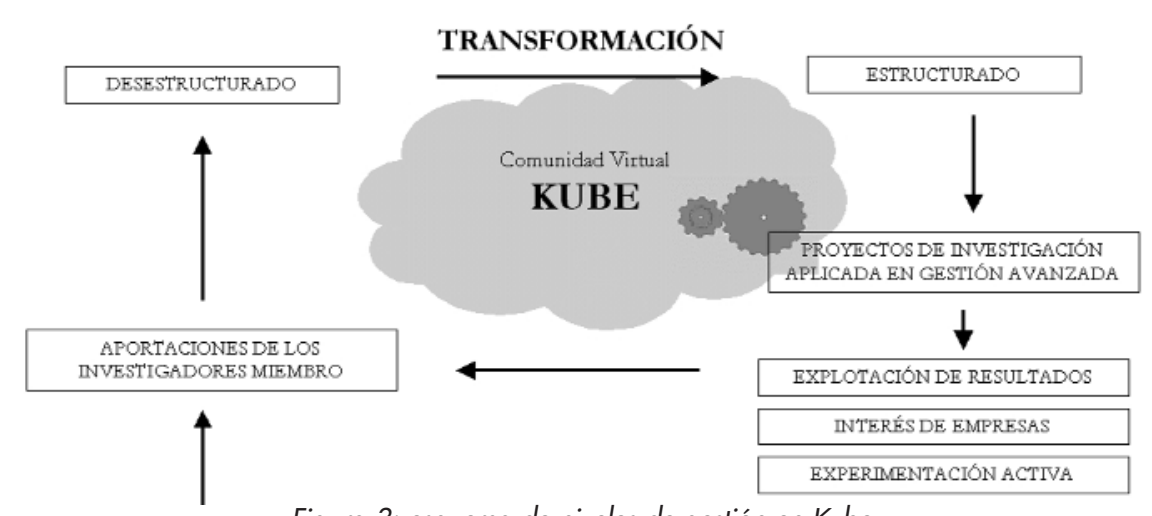

Figura 3: esquema de niveles de gestión en Kube

-Nivel de actividad: permite a los investigadores el desempeño de sus tareas: descarga analítica de horas de trabajo, almacenar o descargar entregables, etc.; traduciéndose todo lo anterior en avances de trabajo dentro del sistema de workflow.

-Nivel operativo: es útil para visualizar una tarea en su globalidad, así como su estado de avance, personas que intervienen, tiempos asignados, presupuesto, incidencias, etc.

-Nivel ejecutivo: el responsable del proyecto puede tener una visión global de todas las tareas que componen el proyecto, acceso a los entregables generados, capacidad de variar los tiempos, el presupuesto, la asignación de personas a alguna tarea, introducir nuevas tareas, etc.

-Nivel estratégico: hace posible tener una imagen global de los proyectos que están siendo llevados a cabo, el grado de implicación de las personas tanto en presupuesto como en horas, grado de avance de cada uno de los proyectos, obtención de estadísticas desglosadas por diferentes conceptos, etc.

Evidentemente estos niveles son acumulativos de tal manera que el último puede realizar las labores propias de cualquiera de los anteriores, más la propia labor es- tratégica y así sucesivamente en sentido descendente.

\section{Gestión por competencias.}

Nos permite coordinar en todo momento la ubicación de nuestros investigadores en los diferentes proyectos así como conocer su grado de disponibilidad, sus competencias, actitudes, aptitudes, etc.

Toda esta información queda reflejada en el árbol de competen- 
cias tanto personales como de la comunidad de investigadores. Dicho árbol es una herramienta dinámica para que cualquier modificación que ocurra en las competencias de un determinado investigador (adquiere experiencia en un área, se involucra en un proyecto concreto, etc.) sea reflejado automáticamente, lo cual mantiene actualizado y facilita la gestión de los recursos humanos de acuerdo a las necesidades de nuevos proyectos.

\section{Robot de búsqueda inteli-} gente.

Otra de las facilidades que presenta Kube es la opción de programar procesos que se ejecutan cada cierto tiempo y según se especifica por el usuario. Una de las aplicaciones de esta funcionalidad es la posibilidad de implementar un sistema de vigilancia de tal manera que el usuario sea notificado cada vez que se modifique alguno de los webs que haya identificado como interesante para su investigación.

Además, nos permite realizar búsquedas internas dentro de Kube sobre múltiples orígenes de datos, tanto si estas fuentes tienen formatos o estructuras diferentes o si se encuentran almacenadas en varias máquinas. Además puede integrar diferentes motores de búsqueda, de los cuales entre los soportados hoy en día tenemos: Verity (Search 97 y K2), Oracle Intermedia y Microsoft Index Server.

\section{Notas}

1. Fuente: Inxight Software, Inc.

2. Actualmente en versión beta desarrollada en MIK S. Coop. por Iker Jaurkikoa.

jaurkikoa@mik.es

3. Versión 1.0 desarrollada por MIK S. Coop., disponible en la dirección:

http://www.mik.es/login.asp

4. Producto perteneciente a la marca $3 M, 1995$ 2003.

Javier Fínez, MIK S. Coop; Emilio González, Ever Documéntica S. A.

finez@mik.es

egonzalez@everdoc.com 